\title{
Effects of the flaxseed lignans secoisolariciresinol diglucoside and its aglycone on serum and hepatic lipids in hyperlipidaemic rats
}

\author{
M. A. Felmlee ${ }^{1}$, G. $\mathrm{Woo}^{2}$, E. Simko ${ }^{3}$, E. S. $\mathrm{Krol}^{4}$, A. D. Muir ${ }^{5}$ and J. Alcorn ${ }^{4} *$ \\ ${ }^{1}$ Department of Pharmaceutical Sciences, University at Buffalo, State University of New York, Buffalo, NY, USA \\ ${ }^{2}$ Leslie Dan Faculty of Pharmacy, University of Toronto, Toronto, ON, Canada \\ ${ }^{3}$ Department of Veterinary Pathology, Western College of Veterinary Medicine, University of Saskatchewan, Saskatoon, \\ SK, Canada \\ ${ }^{4}$ College of Pharmacy and Nutrition, University of Saskatchewan, Saskatoon, SK, Canada \\ ${ }^{5}$ Agriculture and Agri-Food Canada, Saskatoon, SK, Canada \\ (Received 19 June 2008 - Revised 3 November 2008 - Accepted 2 December 2008 - First published online 16 February 2009)
}

The present study involved a comparative analysis of the effects of purified flaxseed lignans, secoisolariciresinol diglucoside (SDG) and its aglycone metabolite (SECO), in hyperlipidaemic rats. For hypercholesterolaemia, female Wistars (six rats per group) were fed a standard or $1 \%$ cholesterol diet and orally administered 0,3 or $6 \mathrm{mg} \mathrm{SDG} / \mathrm{kg}$ or $0,1.6$ or $3.2 \mathrm{mg} \mathrm{SECO} / \mathrm{kg}$ body weight once daily for 4 weeks. Hypertriacylglycerolaemia was induced in male Sprague-Dawley rats (ten rats per group) by supplementing tap water with $10 \%$ fructose. These rats were orally administered 0,3 or $6 \mathrm{mg} \mathrm{SDG} / \mathrm{kg}$ body weight once daily for 2 weeks. Fasting blood samples $(12 \mathrm{~h})$ were collected predose and at the end of the dosing period for serum lipid analyses. Rats were killed and livers rapidly excised and sectioned for lipid, mRNA and histological analyses. Chronic administration of equimolar amounts of SDG and SECO caused similar dose-dependent reductions in rate of body-weight gain and in serum total and LDL-cholesterol levels and hepatic lipid accumulation. SDG and SECO failed to alter hepatic gene expression of commonly reported regulatory targets of lipid homeostasis. SDG had no effect on serum TAG, NEFA, phospholipids and rate of weight gain in $10 \%$ fructose-supplemented rats. In conclusion, our data suggest that the lignan component of flaxseed contributes to the hypocholesterolaemic effects of flaxseed consumption observed in humans. Future studies plan to identify the biochemical mechanism(s) through which flaxseed lignans exert their beneficial effects and the lignan form(s) responsible.

Purified flaxseed lignans: Hyperlipidaemia: Rats

Hypercholesterolaemia and hypertriacylglycerolaemia are independent risk factors of CVD and often occur in association with other CVD risk factors such as obesity, type 2 diabetes mellitus and elevated blood pressure ${ }^{(1)}$. Therapeutic measures designed to reduce blood lipid parameters can decrease the risk for $\mathrm{CVD}^{(1)}$. The need for very safe and effective lipid-lowering therapies directs significant attention toward flaxseed and its various products (for example, ground flaxseed, defatted flaxseed meal, flaxseed extracts) as sources of chemopreventive agents in $\mathrm{CVD}^{(2,3)}$. Recent human clinical trials and pathological animal model investigations with whole flaxseed or flaxseed products (for example, ground flax, flaxseed extracts, lignan-enriched flaxseed product) show clinically favourable changes in blood lipid profiles and other indices of lipid homeostasis with apparent safety $^{(4-8)}$. Unfortunately, such studies cannot definitely ascribe the lipid-lowering effects of flaxseed consumption to any single flaxseed component. Whole flaxseed and its products may contain variable levels of fat (primarily $\alpha$-linolenic acid), dietary fibre and soluble protein as well as lignans, minerals, vitamins and carbohydrates ${ }^{(9)}$, which confound interpretations and probably explain the variable results reported with the use of such materials. Studies using enriched flaxseed lignan sources draw a clearer association between flaxseed lignans and the beneficial lipid-lowering effects of flaxseed $^{(3,10,11)}$. However, optimisation of safety and efficacy of use of a particular chemopreventive therapy require a guarantee of product standardisation. Purified bioactives provide the greatest assurance of quality for determination of safety and efficacy. Yet no study has used purified flaxseed lignan sources for assessments in hypercholesterolaemic and hypertriacylglycerolaemic conditions.

Secoisolariciresinol diglucoside (SDG) is the principal lignan present in flaxseed. Following consumption, SDG is further converted to its aglycone secoisolariciresinol (SECO) and the mammalian lignans enterodiol and enterolactone within the intestine ${ }^{(12)}$. The literature provides little convincing evidence to attribute biological activity to either the plant or mammalian lignans or both. A comparative analysis of individual pure lignans in pathological lipid states is critical

Abbreviations: SDG, secoisolariciresinol diglucoside; SECO, secoisolariciresinol.

* Corresponding author: Dr Jane Alcorn, fax +1 306966 6377, email jane.alcorn@usask.ca 
to confirm which specific lignan(s) contributes to the lipidlowering effects of flaxseed. However, availability of pure lignan is an important barrier to such assessments.

Assurance of lignan safety and efficacy also requires an understanding of their biological mechanism of action. The literature offers several explanations for lignan beneficial health effects, which include direct antioxidant activity ${ }^{(13)}$ and weak oestrogenic or anti-oestrogenic activity ${ }^{(14)}$. Very limited attention addresses potential presystemic mechanisms (i.e. inhibition of cholesterol absorption) or the potential of flaxseed lignans to influence transcriptional regulation of those genes involved in overall hepatic lipid homeostasis. Precedence exists for antihyperlipidaemic effects subsequent to transcriptional regulation of those factors controlling lipid homeostasis $^{(15-18)}$. For example, fibrates ${ }^{(18)}$, statins ${ }^{(16)}$, soya isoflavones ${ }^{(17)}$ and $\mathrm{SDG}^{(3)}$ activate PPAR to mediate the hypolipidaemic effects associated with these compounds. Additionally, sesame lignans alter gene expression of enzymes involved in fatty acid oxidation and synthesis ${ }^{(15)}$. Such examples may identify a similar role for flaxseed lignans in the liver.

A definitive identification of the bioactive lignan form and its underlying mechanism of action are critical to our understanding of flaxseed efficacy and safety in the management of CVD risk factors. The purpose of the present study was to separately administer SDG and its metabolite, SECO, to diet-induced dyslipidaemic rats and compare the extent to which each lignan decreased serum and hepatic lipid levels in hyperlipidaemic rats. In addition, we used quantitative gene expression analysis to provide a preliminary assessment as to whether SDG and/or SECO alter the expression of several regulators of lipid homeostasis to possibly explain their hypolipidaemic effects with chronic oral administration.

\section{Methods}

\section{Animals and chemicals}

Male and female Sprague-Dawley and Wistar rats were obtained from Charles River Canada (St Constant, Quebec) at age 6 or 8 weeks and male Wistar rats $(n 2)$ were obtained from the Animal Resources Centre (University of Saskatchewan, Saskatoon, SK) at age 8 weeks. On the date of arrival, rats were randomly assigned to either control or dietary intervention and according to lignan dose. Rats were housed in groups of two or three or individually (depending on experiment) with controlled temperature $\left(22 \pm 2^{\circ} \mathrm{C}\right)$ and were maintained on a $12 \mathrm{~h}$ light-dark cycle (lights on from 07.00 to 19.00 hours). All rats were fed a standard rodent diet (Prolab ${ }^{\circledR}$ RMH 3000; Purina, Inc., Richmond, IN, USA) (22.5\% protein; $4.5 \%$ fat, ether extract; $6.4 \%$ fat, acid hydrolysis; $4.0 \%$ crude fibre; $6.1 \%$ ash; $1.0 \% \mathrm{Ca} ; 0.75 \%$ $\mathrm{P})$, with or without diet supplementation depending upon the dietary intervention, and provided water ad libitum. Rats were acclimatised to animal handling and oral dosage administration procedures for a period of 1 week before the start of the chronic dosing protocol. All procedures were conducted in accordance with the Canadian Council of Animal Care guidelines for the care and use of laboratory animals and were approved by the Animal Care and Supply Committee of the University of Saskatchewan.
SDG ( $>99 \%$ pure) and SECO ( $>95 \%$ pure) were kind gifts from Agriculture and AgriFood Canada (A. D. M.). RNeasy Midi kits were obtained from Qiagen Inc. (Mississauga, ON, USA) and RNAlater ${ }^{\mathrm{TM}}$ solution was from Sigma-Aldrich (St Louis, MO, USA). For real-time RTPCR we used a Quanti Tect SYBR Green RT-PCR Kit from Qiagen Inc. Commercial kits for total cholesterol, TAG, NEFA, phospholipids and HDL-cholesterol were from Stanbio Laboratory (Boerne, TX, USA) or Wako Chemicals USA, Inc. (Richmond, VA, USA). All other chemicals not otherwise specified were obtained from Sigma-Aldrich.

\section{Dose-dependent effects with diet-induced hypercholesterolaemia study design}

A pilot study was conducted to identify the appropriate rat strain and sex to compare lignan effects on hypercholesterolaemic rats. For the pilot study male and female SpragueDawley $(n 3)$ and female Wistar rats $(n 3)$ at age 6 weeks and male Wistar rats $(n 2)$ at age 8 weeks were evaluated (as indicated for the main study below) and the appropriate sex and strain employed. Our pilot studies showed significant strain and sex differences in serum lipid parameters with SDG administration. After 4 weeks, only female Wistar and Sprague-Dawley control rats demonstrated serum total cholesterol beyond the normal range $(250-700 \mathrm{mg} / \mathrm{l})$, and with SDG administration only female Wistar rats demonstrated distinct reductions in serum total cholesterol $(48 \%)$ and LDL-cholesterol (61\%) (data not shown). Based on the results of the pilot study, female Wistar rats were selected for the subsequent study.

For the main study, upon arrival rats were randomised to either the standard rodent diet or a $1 \%$ cholesterol diet (1\% cholesterol incorporated into Prolab ${ }^{\circledR}$ RMH 3000; Purina, Inc., Richmond, IN, USA). Following the 1-week acclimatisation period, rats on the same diet were randomised to one of six treatment groups ( $n$ 6/group) $(0,3$ or $6 \mathrm{mg} \mathrm{SDG} /$ $\mathrm{kg}$ body weight or $0,1.6$ or $3.2 \mathrm{mg} \mathrm{SECO} / \mathrm{kg}$ body weight). All rats were weighed daily and lignan (SDG or SECO) or vehicle $(0.9 \%$ sodium chloride for SDG; 50:50 0.9\% sodium chloride-polyethylene glycol 400 for SECO) $(0.2 \mathrm{ml})$ was administered orally once daily. After a $12 \mathrm{~h}$ fast blood samples were collected under isoflurane anaesthesia at 0 (pre-dose baseline) and 2 weeks via saphenous venepuncture and at 4 weeks by cardiac puncture into sterilised centrifuge tubes containing no anticoagulant for serum lipid analyses. All blood samples were collected between 09.30 and 10.30 hours. At 4 weeks, rats were euthanised by an overdose of isoflurane anaesthesia and exsanguination. Livers were rapidly excised and sectioned for lipid, RNA and histological analyses. Samples for RNA analyses were stored in RNAla$t e r^{\mathrm{TM}}$ solution at $-20^{\circ} \mathrm{C}$. Segments of liver were snap-frozen in liquid $\mathrm{N}_{2}$ and fixed in $10 \%$ formalin for routine histology.

We assessed food intake (standard diet) in individually housed female Wistars in vehicle control $(n 6)$ and weightmatched SDG-treated ( $n$ 6) rats to determine whether SDG may alter food consumption. Each animal was administered a $0.2 \mathrm{ml}$ volume of vehicle $(0.9 \%$ sodium chloride) or SDG (6 mg SDG/kg body weight, dissolved in $0.9 \%$ sodium chloride) daily by oral administration for 4 weeks. Each animal was weighed daily and food intake was measured every 
other day. SDG did not alter food intake (data not shown) at the highest dosing level.

To assess general lignan safety, blood was collected for haematology and blood chemistry for evaluation of SDG's effect (daily administration of $6 \mathrm{mg} \mathrm{SDG} / \mathrm{kg}$ body weight for 4 weeks) on renal, hepatic and immune systems. Tissues were rapidly excised following euthanasia, weighed and assessed by gross morphological examination. Complete blood chemistry and haematology were performed in the clinical chemistry laboratory at the Royal University Hospital (Saskatoon, Saskatchewan, Canada).

\section{Dose-dependent effects with diet-induced hypertriacylglycerolaemia study design}

For consistency of the model system, initial studies were conducted with female Wistar rats (eight rats per group) obtained at age 6 weeks. We failed to induce hypertriacylglycerolaemia in female Wistars following 4 weeks of $10 \%$ fructose in water. Hence, we used the well-known male SpragueDawley rat model of hypertriacylglycerolaemia. Male Sprague-Dawley rats were housed individually and, after the acclimatisation period, $10 \%$ fructose in water replaced the regular tap water in those groups requiring fructose feeding. Animals were randomly assigned to four treatment groups (ten rats per group): (1) tap water; (2) $10 \%$ fructose in tap water; (3) $10 \%$ fructose in tap water with single daily dose of $3 \mathrm{mg} \mathrm{SDG} / \mathrm{kg}$ body weight; (4) $10 \%$ fructose in tap water with a single daily dose of $6 \mathrm{mg}$ SDG/ $\mathrm{kg}$ body weight. Rats were weighed daily and SDG or vehicle ( $0.9 \%$ sodium chloride) was orally administered once daily within a $2 \mathrm{~h}$ period at the same time each day. Blood samples were collected following a $12 \mathrm{~h}$ fast under isoflurane anaesthesia at 0 (pre-dose baseline) weeks via saphenous venepuncture, and at 2 weeks via cardiac puncture into sterilised centrifuge tubes containing no anticoagulant for serum lipid analyses. All blood samples were collected within a $2 \mathrm{~h}$ period beginning at 06.00 hours. At 2 weeks, rats were euthanised by an overdose of isoflurane anaesthesia and exsanguination. Liver, heart and retroperitoneal adipose tissue were rapidly excised and weighed. Liver samples for RNA analysis were stored in RNAlater ${ }^{\mathrm{TM}}$ solution at $-20^{\circ} \mathrm{C}$. Portions of liver were flash-frozen in liquid $\mathrm{N}_{2}$ and stored in $-80^{\circ} \mathrm{C}$ and fixed in $10 \%$ formalin solution for routine histology.

\section{Analyses of lipids}

Serum total cholesterol, TAG, NEFA, phospholipids and HDL-cholesterol were determined by colorimetric analysis with commercial kits from Stanbio (Boerne, TX, USA) or Wako Chemicals USA, Inc. (Richmond, VA, USA) immediately following serum collection according to the manufacturer directions. LDL-cholesterol levels were determined using the Friedewald method ${ }^{(19)}$.

\section{Histological analyses}

Formalin fixed liver samples were embedded in paraffin, sectioned at $5 \mu \mathrm{m}$, stained with haematoxylin-eosin and examined by light microscopy. Determination of the extent of hepatic lipidosis was performed on oil red O-stained frozen formalin-fixed tissue sections using image analysis software (Northern Eclipse v. 6.0; Empix Imaging, Mississauga, ON, Canada). From each liver sample, four digital images (each representing $3.69 \mathrm{~mm}^{2}$ of hepatic section) were captured by camera (DVC-1310C; Digital Video Camera Company, Austin, TX, USA) using a light microscope (Olympus BH-2; Olympus Optical Co. Ltd, Tokyo, Japan) with a $6.3 \times$ objective lens (Plan 6.3/0.16; Carl Zeiss, Jena, Germany). The area of empty spaces representing empty sinusoids and vessels as well as artifactual tissue separation was selected, determined and subtracted from the total area of the digital image to obtain the total area of hepatic parenchyma (hepatocytes and supportive stroma). The total area of oil red $\mathrm{O}$ staining was selected and determined to calculate the percentage of hepatic parenchyma accumulating oil red $\mathrm{O}$-stained fat. The average percentage of hepatic parenchyma affected by hepatic lipidosis was derived from four digital images for each rat. All hepatic tissue sections were coded and analysed blindly by an American College of Veterinary Pathologists (ACVP) board certified pathologist without knowledge of treatment groups and diets.

\section{RNA analyses}

RNeasy Midi RNA isolation kits were used to isolate total cellular RNA from liver following the manufacturer's protocol. Quantitative real-time RT-PCR analyses were carried out using a SmartCycler ${ }^{\circledR}$ (Cepheid, Sunnyvale, CA, USA) and a Quanti Tect SYBR Green RT-PCR Kit (Qiagen). Primer sequences (Table 1) were designed using Primer3 software (www.broad.mit.edu/cgi-bin/primer/primer3). Sequences, product sizes and optimal annealing temperatures are listed in Table 1. The general real-time RT-PCR protocol for the SmartCycler for analysis of molecular targets is given in the footnotes of Table 1. Gene expression was quantified using the standard curve method for relative quantification.

\section{Statistical analyses}

All statistical analyses were conducted using GraphPad Prism 3.0 (GraphPad, San Diego, CA, USA). All results are expressed as mean values with their standard errors, except liver histological data, which are expressed as medians with their standard errors. Within a lignan treatment, comparisons between treatment groups for each diet were made using one-way ANOVA with a Tukey's post-test. A KruskalWallis test (non-parametric one-way ANOVA) with a Dunn's post-test were used for the analysis of histological data from SDG-treated rats. Non-parametric analysis was employed due to a bimodal distribution in rats treated with $6 \mathrm{mg} \mathrm{SDG} / \mathrm{kg}$ body weight. Comparisons between the lignan administered and treatment group after 4 weeks of lignan administration were made using two-way ANOVA with a Bonferroni post-test. For the food intake study, differences between mean daily food intake and rate of weight gain were tested using an unpaired two-tailed Student's $t$ test. Effects were considered statistically significant when the $P$ value was less than $0 \cdot 05$. 
Table 1. Primer sequences, amplicon sizes and optimisation parameters (annealing temperature and primer efficiency) for real-time RT-PCR analysis of targets important in cholesterol and TAG pathways*

\begin{tabular}{llllrr}
\hline Gene & Accession number & Sense primer & Antisense primer & Product size (bp) & Temperature $\left({ }^{\circ} \mathrm{C}\right)$ \\
\hline ACAT2 & AB075946 & caggacacccagcatcagg & aggatggacagcagcagagc & 162 & 58 \\
ApoE & NM138828 & cgcggtgtgagtgctatcc & acctcctctaggaggtctcg & 179 & 57 \\
$\beta-A c t i n$ & NM031144 & agcgtggctacagcttcacc & tgccacaggattccataccc & 237 & 58 \\
Cyp7a1 & NM012942 & gggcatctcaagcaaacacc & gcactggaaagcctcagagc & 226 & 55 \\
HMGR & X55286 & ggatgcagcacagaatgtgg & acgcccttgaacacctagc & 182 & 57 \\
LDLR & X13722 & cccactcacccaagttcacc & agctgagggatcctccttgc & 176 & 56 \\
PPAR- $\gamma$ & AB011365 & tggggatgtctcacaatgcca & ttcctgtcaagatcgccctcg & 200 & 58 \\
SREBP-2 & XM216989 & cgcaaccagcttcaagtcc & agcgactgtctgcactgtgg & 175 & 57 \\
PPAR- $\alpha$ & M88592 & aaggctatccaggcttgc & gatgtcgcagaatggcttcc & 189 & 57 \\
SREBP-1c & L16995 & gtgtcagcttgtggcagtgg & atgcctcggctatgtgaagg & 236 & 57 \\
\hline
\end{tabular}

ACAT2, acetyl-CoA acetyltransferase 2; Cyp7a1, cytochrome P450 7a1; HMGR, HMG-CoA reductase; LDLR, LDL receptor; SREBP, sterol response element binding protein.

* General real-time RT-PCR protocol for the analysis of mRNA levels: reverse transcription, 30 min at $50^{\circ} \mathrm{C}$; $\mathrm{PCR}$, initial activation step at $95^{\circ} \mathrm{C}$ for 15 min, denaturation at $94^{\circ} \mathrm{C}$ for $15 \mathrm{~s}$, annealing at temperatures indicated above for $30 \mathrm{~s}$, extension at $72^{\circ} \mathrm{C}$ for $30 \mathrm{~s}$.

\section{Results}

\section{Comparison of secoisolariciresinol diglucoside and} secoisolariciresinol in hypercholesterolaemia

SDG $(3.0$ and $6.0 \mathrm{mg} / \mathrm{kg}$ body weight) and SECO $(1.6 \mathrm{mg} / \mathrm{kg}$ body weight) decreased rate of weight gain in standard dietfed female Wistar rats (Table 2). Both low- and high-dose SDG and SECO decreased rate of weight gain in $1 \%$ cholesterol-fed female Wistar rats (Table 3). High-dose SECO also decreased absolute liver weight (Table 2). The differences in weight parameters corresponded to a greater degree of mesenteric fat accumulation and diffuse yellow discolouration of the liver (suggesting hepatic lipidosis) in $1 \%$ cholesterol-fed control rats relative to lignan-treated rats (data not shown). No changes in body weight and mesenteric fat accumulation were observed in rats fed a standard diet treated with either SDG or SECO (data not shown).

Serum lipid parameters after 4 weeks of SDG or SECO treatment remained unchanged in female Wistar rats fed a standard diet (data not shown). In rats fed the $1 \%$ cholesterol diet, 3 and $6 \mathrm{mg} \mathrm{SDG} / \mathrm{kg}$ decreased total cholesterol by 8 and $27 \%$, respectively $(P>0 \cdot 05)$, and LDL-cholesterol by 11 and
$39 \%$, respectively $(P>0.05)$ (Fig. 1). Similarly, 1.6 and $3.2 \mathrm{mg} \mathrm{SECO} / \mathrm{kg}$ decreased total cholesterol by 16 and $15 \%$, respectively $(P>0.05)$, and LDL-cholesterol by 24 and $34 \%$, respectively $(P>0.05)$ (Fig. 1). Equimolar administrations of SDG and SECO resulted in a similar magnitude of reduction in serum total cholesterol and LDL-cholesterol in $1 \%$ cholesterol-fed female Wistar rats.

In rats fed a diet supplemented with $1 \%$ cholesterol, livers exhibited a distinct yellow discolouration on gross examination. This was not observed in rats fed a standard diet with or without SDG or SECO administration. In rats fed the $1 \%$ cholesterol diet, 3 and $6 \mathrm{mg}$ SDG/ $\mathrm{kg}$ decreased hepatic fat accumulation by 8 and $24 \%$, respectively. However, hepatic fat accumulation increased by $7 \%$ with $1.6 \mathrm{mg} / \mathrm{kg}$ SECO, but decreased by $24 \%$ following $3.2 \mathrm{mg} / \mathrm{kg}$ SECO (Fig. 2). Figure 3 illustrates hepatic accumulation of lipids (as visualised by oil red $\mathrm{O}$ staining) in hypercholesterolaemic control rats but a decreased amount of hepatic lipids in rats treated with lignan. The variable degree of hepatic lipidosis had positive and significant correlations $(r>0.44 ; P<0.05)$ with serum lipid parameters of the same rats (data not shown). No other histopathological changes were observed in the livers of these rats.

Table 2. Body and liver weight indices for female Wistar rats ( $n 6$ per treatment group) fed standard rat chow ( 5 weeks) and treated with oral doses of $0.0,3.0$ or $6.0 \mathrm{mg}$ secoisolariciresinol diglucoside (SDG) $/ \mathrm{kg}$ body weight (BW) or $0.0,1.6$ or $3.2 \mathrm{mg}$ secoisolariciresinol (SECO)/kg BW once daily (same time of day) for 4 weeks§॥

(Mean values with their standard errors)

\begin{tabular}{|c|c|c|c|c|c|c|c|c|c|c|c|c|}
\hline \multirow[b]{3}{*}{ Weight parameter } & \multicolumn{6}{|c|}{$\mathrm{SDG}(\mathrm{mg} / \mathrm{kg} \mathrm{BW})$} & \multicolumn{6}{|c|}{ SECO (mg/kg BW) } \\
\hline & \multicolumn{2}{|c|}{0.0} & \multicolumn{2}{|c|}{$3 \cdot 0$} & \multicolumn{2}{|c|}{$6 \cdot 0$} & \multicolumn{2}{|c|}{$0 \cdot 0$} & \multicolumn{2}{|c|}{$1 \cdot 6$} & \multicolumn{2}{|c|}{$3 \cdot 2$} \\
\hline & Mean & SEM & Mean & SEM & Mean & SEM & Mean & SEM & Mean & SEM & Mean & SEM \\
\hline Final BW (g) & 250 & $5 \cdot 0$ & 247 & 7.9 & $228^{*}$ & 1.8 & 257 & 10 & 263 & 5.9 & 251 & $6 \cdot 8$ \\
\hline Absolute weight gain (g) & $47 \cdot 5$ & 4.4 & 47.5 & 4.0 & $36 \cdot 0$ & $3 \cdot 8$ & $87 \cdot 3$ & $5 \cdot 1$ & $88 \cdot 2$ & $5 \cdot 2$ & $97 \cdot 8$ & $6 \cdot 1$ \\
\hline Rate of weight gain $(\mathrm{g} / \mathrm{d})$ & 1.76 & 0.035 & $1.43^{*}$ & 0.006 & $1 \cdot 28^{\star} \dagger$ & 0.029 & 3.25 & 0.07 & 3.04 * & 0.046 & $3 \cdot 29^{\star} \ddagger$ & 0.056 \\
\hline Liver weight (g) & $7 \cdot 38$ & 0.43 & $7 \cdot 17$ & 0.42 & 6.47 & 0.34 & 8.43 & 0.61 & 8.60 & 0.49 & 8.45 & 0.23 \\
\hline Liver weight/BW (g/kg) & 29.4 & 1.4 & 29.0 & 1.2 & $28 \cdot 3$ & 1.5 & $32 \cdot 7$ & 1.3 & $32 \cdot 6$ & 1.5 & 33.8 & 1.4 \\
\hline
\end{tabular}

* Mean value was significantly different from that of the control group $(P<0.05)$.

† Mean value was significantly different from that of the group that received SDG at $3.0 \mathrm{mg} / \mathrm{kg} \mathrm{BW}(P<0.05)$.

$\ddagger$ Mean value was significantly different from that of the group that received SECO at $1.6 \mathrm{mg} / \mathrm{kg} \mathrm{BW}(P<0.05)$.

$\S$ Data were analysed with one-way ANOVA with Tukey's post-test.

\|On a molar basis the doses are equivalent to $0.0,4.4$ and $8.8 \mu \mathrm{mol} / \mathrm{kg} \mathrm{BW}$. 
Table 3. Body and liver weight indices for female Wistar rats ( $n 6$ per treatment group) fed a $1 \%$ cholesterol diet (5 weeks) and treated with oral doses of $0.0,3.0$ or $6.0 \mathrm{mg}$ secoisolariciresinol diglucoside (SDG)/kg body weight (BW) or $0.0,1.6$ or $3.2 \mathrm{mg}$ secoisolariciresinol (SECO)/kg BW once daily (same time of day) for 4 weeks $¥ \S$

(Mean values with their standard errors)

\begin{tabular}{|c|c|c|c|c|c|c|c|c|c|c|c|c|}
\hline \multirow[b]{3}{*}{ Weight parameter } & \multicolumn{6}{|c|}{ SDG (mg/kg BW) } & \multicolumn{6}{|c|}{ SECO (mg/kg BW) } \\
\hline & \multicolumn{2}{|c|}{0.0} & \multicolumn{2}{|c|}{$3 \cdot 0$} & \multicolumn{2}{|c|}{$6 \cdot 0$} & \multicolumn{2}{|c|}{0.0} & \multicolumn{2}{|c|}{$1 \cdot 6$} & \multicolumn{2}{|c|}{$3 \cdot 2$} \\
\hline & Mean & SEM & Mean & SEM & Mean & SEM & Mean & SEM & Mean & SEM & Mean & SEM \\
\hline Final BW (g) & 269 & 4.9 & 260 & 4.4 & 257 & $3 \cdot 7$ & 277 & 11 & 271 & $6 \cdot 9$ & 261 & $4 \cdot 1$ \\
\hline Absolute weight gain (g) & $59 \cdot 0$ & $6 \cdot 7$ & 55.5 & $5 \cdot 1$ & $57 \cdot 2$ & 0.60 & 110 & $5 \cdot 8$ & 103 & $3 \cdot 2$ & 102 & $2 \cdot 9$ \\
\hline Rate of weight gain $(\mathrm{g} / \mathrm{d})$ & $2 \cdot 06$ & 0.080 & $1.96^{*}$ & 0.03 & $1.95^{*}$ & 0.040 & 4.01 & 0.08 & $3.54 *$ & 0.060 & $3.63 \star \dagger$ & 0.040 \\
\hline Liver weight $(\mathrm{g})$ & $9 \cdot 25$ & 0.35 & 8.37 & 0.16 & $8 \cdot 15$ & 0.44 & $11 \cdot 0$ & 0.36 & 9.52 & 0.57 & $9 \cdot 33^{*}$ & 0.22 \\
\hline Liver weight/BW (g/kg) & $34 \cdot 2$ & 1.6 & $32 \cdot 3$ & 0.88 & $31 \cdot 6$ & 1.4 & $39 \cdot 7$ & $0 \cdot 86$ & $35 \cdot 2$ & $2 \cdot 3$ & $35 \cdot 7$ & 0.30 \\
\hline
\end{tabular}

* Mean value was significantly different from that of the control group $(P<0.05)$.

† Mean value was significantly different from that of the group that received SECO at $1.6 \mathrm{mg} / \mathrm{kg} \mathrm{BW}(P<0.05)$.

$\ddagger$ Data were analysed with one-way ANOVA with Tukey's post-test.

$\S$ On a molar basis the doses are equivalent to $0.0,4.4$ and $8.8 \mu \mathrm{mol} / \mathrm{kg} \mathrm{BW}$.

Hepatic enzymes and proteins involved in cholesterol metabolic and uptake pathways were selected for analysis based on significant ability to regulate serum lipid levels as identified in the literature. For hypercholesterolaemia the targets included transcription factors (sterol response element binding protein (SREBP)-2 and PPAR- $\gamma$ ), the cholesterol metabolic enzymes (HMG-CoA reductase, cytochrome P450 7a1 (Cyp7a1) and acetyl-CoA acetyltransferase 2 (ACAT2)) and proteins involved in cholesterol hepatic uptake and transport (LDL

(A)

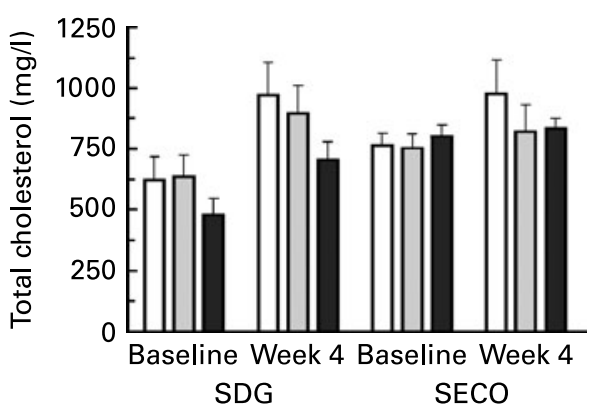

(C)

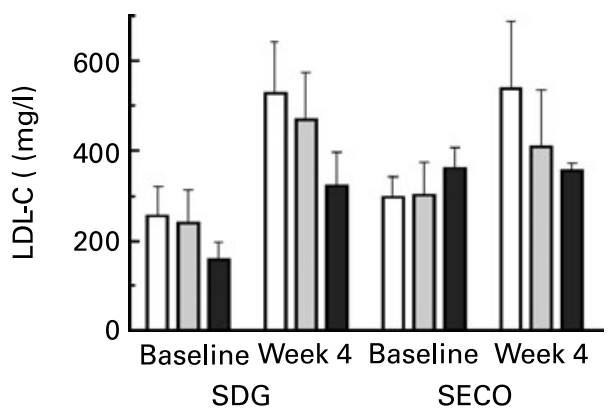

receptor and apoE). SDG administration to rats fed a diet supplemented with $1 \%$ cholesterol resulted in a 54 and $66 \%$ increase $(P<0.05)$ in ACAT2 expression at 3 and $6 \mathrm{mg} / \mathrm{kg}$, respectively (Table 4$)$. No statistically significant changes in gene expression were observed for the other targets. Following the $3.2 \mathrm{mg} \quad \mathrm{SECO} / \mathrm{kg}$ dosing administration, Cyp7a1 expression decreased by $72 \%(P<0 \cdot 05)$. No other significant changes in expression levels were observed for the other targets (Table 4).
(B)

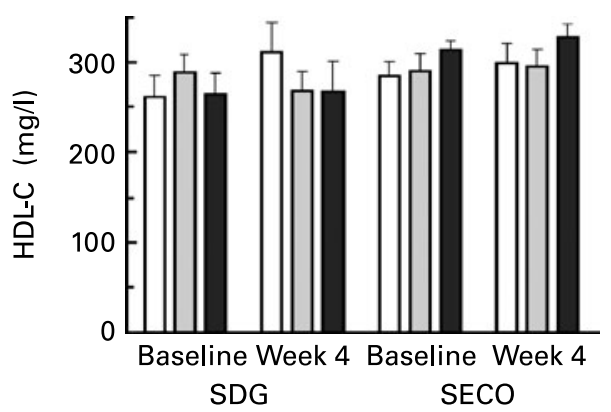

(D)

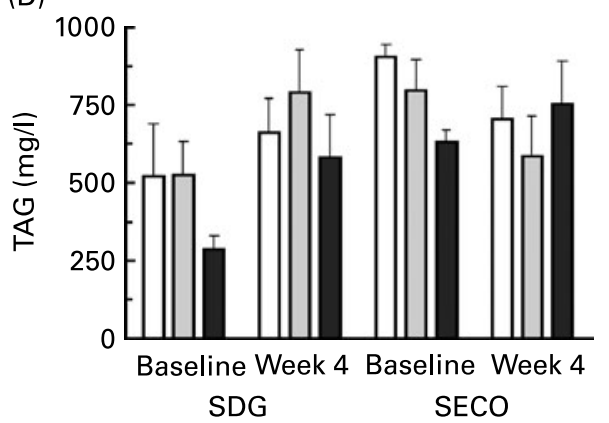

Fig. 1. Serum lipid parameters (total cholesterol (A), HDL-cholesterol (HDL-C) (B), LDL-cholesterol (LDL-C) (C) and TAG (D)) following chronic daily oral secoisolariciresinol diglucoside (SDG) or secoisolariciresinol (SECO) administration in female Wistar rats fed a $1 \%$ cholesterol diet for 1 week before initiation of SDG or SECO dosing and during the lignan dosing interval (4 weeks). Rats ( $n$ 6) were randomly assigned to 0.0 ( $\square$ ), 3.0 ( $\square$ ) or $6.0(\square)$ mg SDG/kg body weight or 0.0 (口), $1.6(\square)$ or $3.2(\square) \mathrm{mg} \mathrm{SECO} / \mathrm{kg}$ body weight. (Note that on a molar basis the doses are equivalent to $0,4.4$ and $8.8 \mu \mathrm{mol} / \mathrm{kg}$ body weight.). SDG or SECO was administered orally once daily (same time of day). Blood was collected from the same animal following an overnight fast via saphenous venepuncture under isoflurane anaesthesia just before initiation of dosing (baseline) and then again at 4 weeks. Values are means, with their standard errors represented by vertical bars. 


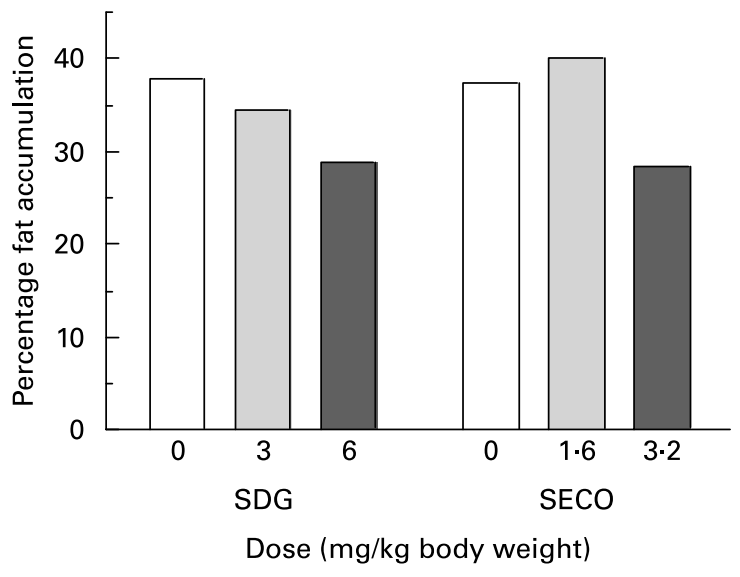

Fig. 2. Median percentage fat accumulation $(n 6)$ in hepatic parenchyma of female Wistar rats fed a $1 \%$ cholesterol diet for 5 weeks. Rats were randomly assigned to $0.0,3.0$ or $6.0 \mathrm{mg}$ secoisolariciresinol diglucoside $(\mathrm{SDG}) / \mathrm{kg}$ body weight or $0.0,1.6$ or $3.2 \mathrm{mg}$ secoisolariciresinol $(\mathrm{SECO}) / \mathrm{kg}$ body weight administered orally once daily for 4 weeks. (Note that on a molar basis the doses are equivalent to $0,4.4$ and $8.8 \mu \mathrm{mol} / \mathrm{kg}$ body weight.) Histological evaluation of oil red O-stained liver slices involved a semi-quantitative blinded analysis and fat accumulation was measured by digital analysis of stained liver sections.

\section{Effects of secoisolariciresinol diglucoside in} hypertriacylglycerolaemia

SDG had no effect on the weight parameters of male Sprague-Dawley rats supplemented with $10 \%$ fructose in tap water relative to controls (Table 5). Analysis of serum TAG, NEFA and phospholipids showed that 2 weeks of supplementation with $10 \%$ fructose in water induced hypertriacylglycerolaemia in male Sprague-Dawley rats (data not shown). However, no statistically significant changes in serum TAG, phospholipids or NEFA were observed with SDG administration relative to non-supplemented rats (data not shown). In rats supplemented with $10 \%$ fructose in tap water, livers exhibited a distinct yellow discolouration on gross examination, but SDG had no effect on hepatic lipids (data not shown). The $10 \%$ fructose in water supplementation resulted in an expected down-regulation of PPAR- $\alpha^{(20)}$ and up-regulation of SREBP-1c $\mathrm{c}^{(21)}$, but SDG administration had no impact on expression levels relative to $10 \%$ fructose in water-supplemented controls (Table 6).

\section{Safety of orally administered secoisolariciresinol diglucoside}

Daily oral administration of SDG (3 mg/kg body weight) for 4 weeks caused no adverse health effects or changes in body or organ weights in female Wistar juvenile rats (data not shown). Haematology and blood chemistry analysis also showed no changes, except in the erythrocyte distribution width (data not shown). However, the values for erythrocyte distribution width in both groups were within the normal range.

\section{Discussion}

The present study represents the first comparative analysis of the effects of purified flaxseed plant lignans in hyperlipidaemic rat models. Many studies have investigated the lipid-lowering effects of whole flaxseed or flaxseed products ${ }^{(4-7,11,22)}$, and one study reported the effects of purified SDG on serum lipid parameters in high-fat diet-induced obese mice ${ }^{(3)}$. In the present study, we evaluated several indices of lipid homeostasis in diet-induced hypercholesterolaemic and hypertriacylglycerolaemic rat models, including serum lipid parameters, body and liver weight, steatosis, and transcriptional changes in commonly reported targets of hepatic lipid homeostasis. We assessed these indices to compare the activity of purified SDG $v$. its aglycone metabolite, SECO, on lipid homeostasis and to glean some preliminary information on possible mechanisms of action. Our data suggest that chronic
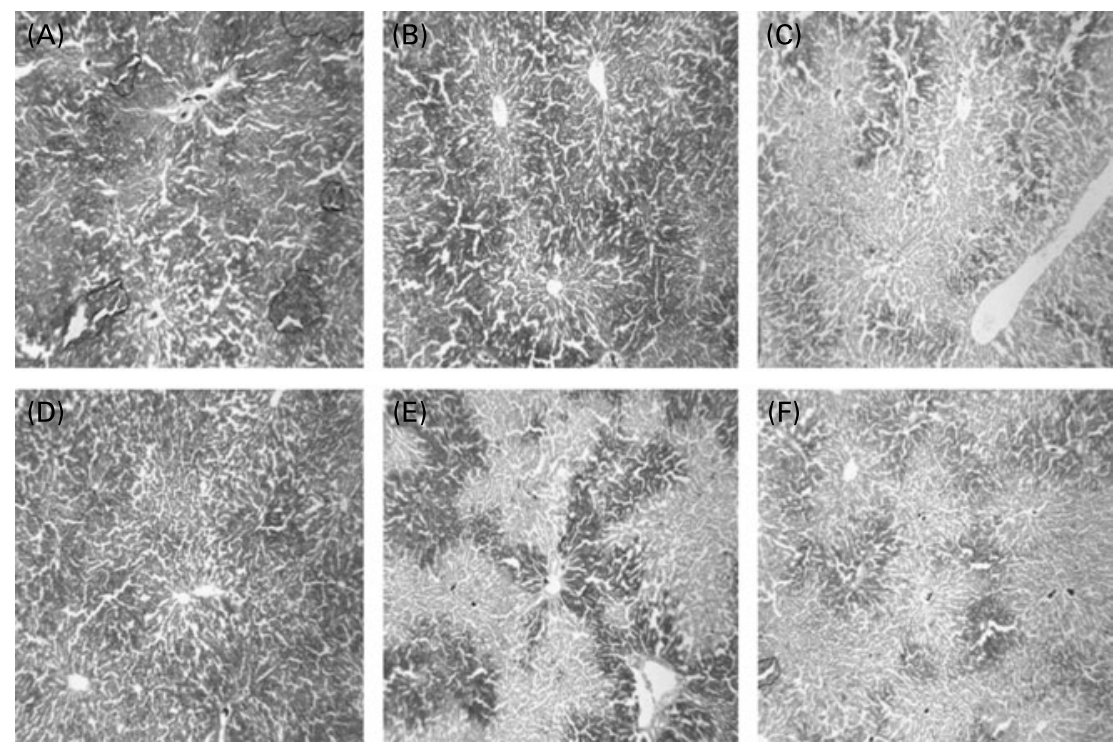

Fig. 3. Oil red O-stained frozen hepatic tissue sections to identify the extent of hepatic lipidosis. Female Wistar rats $(n 6)$ were fed a $1 \%$ cholesterol diet and randomly assigned to $0.0,3.0$ or $6.0 \mathrm{mg}$ secoisolariciresinol diglucoside (SDG)/kg body weight (A, B or C, respectively) or $0.0,1.6$ or $3.2 \mathrm{mg}$ secoisolariciresinol (SECO)/kg body weight (D, E or F, respectively). 
Table 4. Percentage changes in hepatic mRNA expression levels of key genes involved in cholesterol metabolism in female Wistar rats $(n 6)$ fed a $1 \%$ cholesterol diet and treated with daily oral administration of secoisolariciresinol diglucoside (SDG) or secoisolariciresinol (SECO)†

\begin{tabular}{|c|c|c|c|c|}
\hline \multirow[b]{2}{*}{ Gene } & \multicolumn{2}{|c|}{$\mathrm{SDG}(\mathrm{mg} / \mathrm{kg} \mathrm{BW})$} & \multicolumn{2}{|c|}{ SECO (mg/kg BW) } \\
\hline & 3.0 & $6 \cdot 0$ & 1.6 & $3 \cdot 2$ \\
\hline АCAT2 & $\uparrow 54 \%^{*}$ & $\uparrow 66 \% \%^{*}$ & $0 \%$ & $\downarrow 7 \%$ \\
\hline ApoE & $\downarrow 35 \%$ & $\downarrow 21 \%$ & $\uparrow 35 \%$ & $\downarrow 0.6 \%$ \\
\hline Сур7a1 & $\downarrow 7 \%$ & $\uparrow 10 \%$ & $\downarrow 36 \%$ & $\downarrow 71 \% *$ \\
\hline HMGR & $\uparrow 27.5 \%$ & $\uparrow 35 \%$ & $\uparrow 8 \%$ & $\downarrow 6 \%$ \\
\hline LDLR & $\downarrow 32 \%$ & $\uparrow 6 \%$ & $\downarrow 6 \%$ & $\downarrow 24 \%$ \\
\hline PPAR- $\gamma$ & $\downarrow 14 \%$ & $\uparrow 2 \%$ & $\uparrow 17 \%$ & $\uparrow 17 \%$ \\
\hline SREBP-2 & $\downarrow 27 \%$ & $\downarrow 19 \%$ & $\downarrow 15 \%$ & $\downarrow 22 \%$ \\
\hline
\end{tabular}

BW, body weight; ACAT2, acetyl-CoA acetyltransferase 2; Cyp7a1, cytochrome P450 7a1; HMGR, HMG-CoA reductase; LDLR, LDL receptor; SREBP-2, sterol response element binding protein-2.

${ }^{*}$ Change was significantly different from that of the control group $(P<0.05)$.

† Rats were randomly assigned to $0.0,3.0$ or $6.0 \mathrm{mg} \mathrm{SDG} / \mathrm{kg} \mathrm{BW}$ or $0.0,1.6$ or $3.2 \mathrm{mg} \mathrm{SECO} / \mathrm{kg}$ BW dose groups and mRNA expression was assessed after 4 weeks using real-time reverse transcription-PCR.

administration of equimolar levels of SDG and SECO cause similar dose-dependent reductions in serum and hepatic cholesterol levels, but neither SDG nor SECO caused significant changes in hepatic gene expression of lipid homeostasis targets. Furthermore, SDG had no effect on serum TAG in our model of hypertriacylglycerolaemia.

We observed a dose-related decrease $(P>0.05)$ in total and LDL-cholesterol in rats supplemented with $1 \%$ cholesterol in their diet and dosed daily with SDG and SECO. SDG and SECO also decreased rate of weight gain and hepatic parenchymal fat accumulation in rats fed the high-cholesterol diet. These data provide supportive evidence that flaxseed lignans contribute to the serum and hepatic cholesterol-lowering effects of flaxseed observed in humans and in animal studies. Interestingly, similar reductions in serum total cholesterol and LDL-cholesterol (31 and $32 \%$, respectively) in a diet-induced hypercholesterolaemic rabbit model ${ }^{(23)}$ required 2.5 times higher SDG doses than the dose used in the present study. This may suggest that: (1) SDG exerts a greater cholesterollowering effect in rats; (2) significant interspecies differences exist in the pharmacokinetics of flaxseed lignans; and/or (3) the effects of SDG may plateau with increasing dose. Although comparisons between the hypocholesterolaemic effects of purified lignans or lignan-enriched flaxseed products with other flaxseed products are difficult, the enriched lignan products generally produce greater reductions in serum lipid parameters than reported in the literature. Recently, significant reductions in serum cholesterol levels in hypercholesterolaemic patients administered a lignan-enriched (35\% lignan) flaxseed polymer was reported ${ }^{(11)}$, which exceeded reductions reported with other flaxseed products. One would expect to observe smaller reductions in serum lipid levels with lignanenriched flaxseed products if the hypocholesterolaemic effects involved a synergism between two or more components of flaxseed. Our data support a role for the lignan component in mediating the hypocholesterolaemic effects associated with flaxseed consumption ${ }^{(11)}$.

Carbohydrate-rich diets are known to stimulate lipogenesis in both the liver and adipose tissue to cause increased plasma and hepatic TAG levels ${ }^{(24)}$. Supplementation with $10 \%$ fructose in tap water effectively induces hypertriacylglycerolaemia in male rats ${ }^{(20)}$. SDG administration at either dosing level caused no significant changes in body and organ weights, serum TAG, phospholipids and NEFA, and hepatic steatosis relative to vehicle control fructose-fed rats. Given the similar dose-response effects of SDG and SECO in our high-cholesterol diet-fed rats and limited availability of purified SECO, we omitted SECO administration in this model of hypertriacylglycerolaemia. Interestingly, administration of flaxseed products in dyslipidaemic models reported in the literature has shown variable effects on serum TAG levels ${ }^{(6,14,23,25,26)}$. Human clinical trials also report variable effects of flaxseed consumption on serum $\mathrm{TAG}^{(2,7,8,22,27,28)}$. The present study contributes to this lack of consensus. Interestingly, a close structural analogue of SECO, masoprocol (nordihydroguaiaretic acid), significantly decreased serum TAG levels in a rat model of hypertriacylglycerolaemia ${ }^{(29)}$. This offers intriguing evidence for a possible effect of SECO on TAG homeostasis and may depend upon the species, strain, age, sex and method of dietary manipulation used to induce hypertriacylglycerolaemic states.

Table 5. Weight indices for male Sprague-Dawley rats ( $n 10$ per treatment group) supplemented with 0 or $10 \%$ fructose in tap water and treated with oral doses of $0.0,3.0$ or $6.0 \mathrm{mg}$ secoisolariciresinol diglucoside (SDG)/kg body weight (BW) once daily (same time of day) for 2 weeks †‡

(Mean values with their standard errors)

\begin{tabular}{|c|c|c|c|c|c|c|c|c|}
\hline \multirow[b]{3}{*}{ Weight parameter } & & & \multicolumn{6}{|c|}{$10 \%$ Fructose + SDG (mg/kg BW) } \\
\hline & \multicolumn{2}{|c|}{$\begin{array}{c}\text { No fructose and } \\
\text { no SDG }\end{array}$} & \multicolumn{2}{|c|}{0.0} & \multicolumn{2}{|c|}{$3 \cdot 0$} & \multicolumn{2}{|c|}{$6 \cdot 0$} \\
\hline & Mean & SEM & Mean & SEM & Mean & SEM & Mean & SEM \\
\hline Final BW (g) & 281 & $6 \cdot 3$ & 306 & $7 \cdot 6$ & 285 & $5 \cdot 9$ & 296 & $7 \cdot 9$ \\
\hline Absolute weight gain (g) & $91 \cdot 0$ & 13 & 100 & 38 & $83 \cdot 0$ & 32 & 106 & 20 \\
\hline Rate of weight gain $(\mathrm{g} / \mathrm{d})$ & 6.90 & 0.080 & 8.40 & $0 \cdot 10$ & $6 \cdot 90$ & 0.10 & $7 \cdot 90$ & $0 \cdot 10$ \\
\hline Liver weight $(\mathrm{g})$ & $8 \cdot 80$ & 0.30 & $16 \cdot 1^{*}$ & 0.83 & $15 \cdot 2^{*}$ & 0.65 & $14 \cdot 6^{*}$ & 0.92 \\
\hline Liver weight/BW (g/kg) & 31.6 & 0.74 & $52 \cdot 7^{*}$ & $2 \cdot 4$ & $53 \cdot 4^{*}$ & 1.9 & $49 \cdot 1^{*}$ & $2 \cdot 1$ \\
\hline Retroperitoneal adipose weight (g) & $2 \cdot 27$ & 0.18 & $4 \cdot 51^{*}$ & 0.61 & 3.63 & 0.36 & 3.68 & 0.35 \\
\hline
\end{tabular}

* Mean value was significantly different from that of the no-fructose, no-SDG control group $(P<0.05)$.

† Data were analysed with one-way ANOVA with Tukey's post-test.

$\ddagger$ On a molar basis the doses are equivalent to $0.0,4.4$ and $8.8 \mu \mathrm{mol} / \mathrm{kg} \mathrm{BW}$. 
Table 6. Hepatic mRNA expression levels (expressed as percentage of non-fructose-supplemented control) of key transcription factors involved in hepatic TAG metabolism in male Sprague-Dawley rats $(n 10)$ with or without supplementation of $10 \%$ fructose in tap water and treated with $0.0,3.0$ or $6.0 \mathrm{mg}$ secoisolariciresinol diglucoside (SDG) $/ \mathrm{kg}$ body weight (BW) after 2 weeks using real-time RT-PCR

\begin{tabular}{lcccc} 
& & \multicolumn{2}{c}{$\begin{array}{c}10 \% \text { Fructose in water + } \\
\text { SDG }(\mathrm{mg} / \mathrm{kg} \mathrm{BW})\end{array}$} \\
\cline { 3 - 5 } Gene & No fructose and no SDG & 0.0 & 3.0 & $6 \cdot 0$ \\
\hline PPAR- $\alpha$ & 100 & $66 \cdot 1$ & 89.9 & 86.6 \\
SREBP-1c & 100 & 1640 & 1490 & 1010
\end{tabular}

SREBP-1c, sterol response element binding protein-1c.

We observed almost no statistically significant changes in hepatic gene expression following SDG or SECO administration. We evaluated targets that represented major regulatory control points in cholesterol or TAG homeostasis in the liver. The lack of lignan affect on the expression levels of these targets suggests that lignans act through other primary mechanism(s) not yet identified. Nevertheless, SDG and SECO seem to act with apparent safety at pharmacologically relevant doses, as daily SDG administration for 4 weeks resulted in no gross morphological changes or changes in body and organ weights, and no obvious toxicity to the liver, kidney and haematopoietic systems. These findings confirm the general safety of flaxseed lignans noted in the literature ${ }^{(11,30)}$.

In conclusion, our data provide supportive evidence that the lignan component of flaxseed contributes to the hypocholesterolaemic effects of flaxseed consumption observed in humans and animals. SDG and SECO caused similar dose-dependent reductions in serum and hepatic cholesterol profiles in highcholesterol diet-fed rats, but had no effect on TAG levels in high-carbohydrate diet-fed rats. More studies are necessary to identify the exact biochemical mechanism(s) through which flaxseed lignans exert their beneficial effects and the exact identity of the active lignan form (i.e. plant or mammalian). Importantly, the pharmacokinetics of flaxseed lignans remain poorly understood, yet the biological activity and potential toxicity of lignans in vivo depend upon their pharmacokinetic characteristics. Identification of the mechanism through which flaxseed lignans improve lipid parameters, an understanding of lignan pharmacokinetics, and additional investigations into the comparative efficacy and safety of plant and mammalian lignans may lead to the emergence of flaxseed, or its purified lignan forms, as an invaluable therapeutic strategy in the management of hyperlipidaemic patients.

\section{Acknowledgements}

The present study was funded in part by the Saskatchewan Health Research Foundation and in part by an internal College of Pharmacy and Nutrition grant. All authors contributed written sections to the manuscript. M. A. F. and G. W. conducted the experimental work; E S. conducted the histological analysis; A. D. M. provided the purified lignans; E. K. provided financial support to M. A. F. and to the experimental work;
J. A. provided financial support to G. W. and to the experimental work and provided overall direction to the research.

No conflict of interest exists.

\section{References}

1. Bestermann W, Houston MC, Basile J, et al. (2005) Addressing the global cardiovascular risk of hypertension, dyslipidemia, diabetes mellitus, and the metabolic syndrome in the southeastern United States, part II: treatment recommendations for management of the global cardiovascular risk of hypertension, dyslipidemia, diabetes mellitus, and the metabolic syndrome. Am J Med Sci 329, 292-305.

2. Bloedon LT \& Szapary PO (2004) Flaxseed and cardiovascular risk. Nutr Rev 62, 18-27.

3. Fukumitsu S, Aida K, Ueno N, et al. (2008) Flaxseed lignan attenuates high-fat diet-induced fat accumulation and induces adiponectin expression in mice. Br J Nutr 100, 669-676.

4. Prasad K (2007) A study on regression of hypercholesterolemic atherosclerosis in rabbits by flax lignan complex. J Cardiovasc Pharmacol Ther 12, 304-313.

5. Ratnayake WM \& Gilani GS (2003) Protective effects of flax meal against hypercholesterolemia and hypertriglyceridemia in rats. J Am Coll Nutr 22, 157-164.

6. Prasad K (2005) Hypocholesterolemic and antiatherosclerotic effect of flax lignan complex isolated from flaxseed. Atherosclerosis 179, 269-275.

7. Jenkins DJ, Kendall CW, Vidgen E, et al. (1999) Health aspects of partially defatted flaxseed, including effects on serum lipids, oxidative measures, and ex vivo androgen and progestin activity: a controlled crossover trial. Am J Clin Nutr 69, 395-402.

8. Lucas EA, Wild RD, Hammond LJ, et al. (2002) Flaxseed improves lipid profile without altering biomarkers of bone metabolism in postmenopausal women. J Clin Endocrinol Metab 87, 1527-1532.

9. Hall C III \& Tulbek MC Xu Y (2006) Flaxseed. Adv Food Nutr Res 51, 1-97.

10. Pan A, Sun J, Chen Y, et al. (2007) Effects of a flaxseed-derived lignan supplement in type 2 diabetic patients: a randomized, double-blind, cross-over trial. PLOS ONE 2, e1148.

11. Zhang W, Wang X, Liu Y, et al. (2008) Dietary flaxseed lignan extract lowers plasma cholesterol and glucose concentrations in hypercholesterolaemic subjects. Br J Nutr 99, 1301-1309.

12. Axelson M, Sjovall J, Gustafsson BE, et al. (1982) Origin of lignans in mammals and identification of a precursor from plants. Nature 298, 659-660.

13. Hu C, Yuan YV \& Kitts DD (2007) Antioxidant activities of the flaxseed lignan secoisolariciresinol diglucoside, its aglycone secoisolariciresinol and the mammalian lignans enterodiol and enterolactone in vitro. Food Chem Toxicol 45, 2219-2227.

14. Bhathena SJ \& Velasquez MT (2002) Beneficial role of dietary phytoestrogens in obesity and diabetes. Am J Clin Nutr 76, $1191-1201$.

15. Kushiro M, Masaoka T, Hageshita S, et al. (2002) Comparative effect of sesamin and episesamin on the activity and gene expression of enzymes in fatty acid oxidation and synthesis in rat liver. J Nutr Biochem 13, 289-295.

16. Martin G, Duez H, Blanquart C, et al. (2001) Statin-induced inhibition of the Rho-signaling pathway activates PPAR $\alpha$ and induces HDL apoA-I. J Clin Invest 107, 1423-1432.

17. Mezei O, Banz WJ, Steger RW, et al. (2003) Soy isoflavones exert antidiabetic and hypolipidemic effects through the PPAR pathways in obese Zucker rats and murine RAW 264.7 cells. J Nutr 133, 1238-1243. 
18. Schoonjans K, Staels B \& Auwerx J (1996) Role of the peroxisome proliferator-activated receptor (PPAR) in mediating the effects of fibrates and fatty acids on gene expression. $J$ Lipid Res 37, 907-925.

19. Friedewald WT, Levy RI \& Fredrickson DS (1972) Estimation of the concentration of low-density lipoprotein cholesterol in plasma, without use of the preparative ultracentrifuge. Clin Chem 18, 499-502.

20. Roglans N, Sanguino E, Peris C, et al. (2002) Atorvastatin treatment induced peroxisome proliferator-activated receptor $\alpha$ expression and decreased plasma nonesterified fatty acids and liver triglyceride in fructose-fed rats. J Pharmacol Exp Ther 302, 232-239.

21. Shimomura I, Hammer RE, Richardson JA, et al. (1998) Insulin resistance and diabetes mellitus in transgenic mice expressing nuclear SREBP-1c in adipose tissue: model for congenital generalized lipodystrophy. Genes Dev 12, 3182-3194.

22. Bierenbaum ML, Reichstein R \& Watkins TR (1993) Reducing atherogenic risk in hyperlipemic humans with flax seed supplementation: a preliminary report. J Am Coll Nutr 12, 501-504.

23. Prasad K (1999) Reduction of serum cholesterol and hypercholesterolemic atherosclerosis in rabbits by secoisolariciresinol diglucoside isolated from flaxseed. Circulation 99, 1355-1362.
24. Kersten S (2001) Mechanisms of nutritional and hormonal regulation of lipogenesis. EMBO Rep 2, 282-286.

25. Bhathena SJ, Ali AA, Haudenschild C, et al. (2003) Dietary flaxseed meal is more protective than soy protein concentrate against hypertriglyceridemia and steatosis of the liver in an animal model of obesity. J Am Coll Nutr 22, 157-164.

26. Prasad K, Mantha SV, Muir AD, et al. (1998) Reduction of hypercholesterolemic atherosclerosis by CDC-flaxseed with very low $\alpha$-linolenic acid. Atherosclerosis 136, 367-375.

27. Lemay A, Dodin S, Kadri N, et al. (2002) Flaxseed dietary supplement versus hormone replacement therapy in hypercholesterolemic menopausal women. Obstet Gynecol 100, 495-504.

28. Cunnane SC, Ganguli S, Menard C, et al. (1993) High $\alpha$-linolenic acid flaxseed (Linum usitatissimum): some nutritional properties in humans. Br J Nutr 69, 443-453.

29. Gowri MS, Reaven GM \& Azhar S (1999) Masoprocol lowers blood pressure in rats with fructose-induced hypertension. Am J Hypertens 12, 744-746.

30. Hemmings SJ \& Barker L (2004) The effects of dietary flaxseed on the Fischer 344 rat: I. Development, behaviour, toxicity and the activity of liver $\gamma$-glutamyltranspeptidase. Cell Biochem Funct 22, 113-121. 